\title{
Pneumolysin generates multiple conductance pores in the membrane of nucleated cells
}

Rana G. El-Rachkidy ${ }^{\mathrm{a}, ~ *}$, Noel W. Davies ${ }^{\mathrm{b}}$ and Peter W. Andrew ${ }^{\mathrm{a}}$

${ }^{a}$ Department of Infection, Immunity and Inflammation, University of Leicester, University Road, Leicester, LE1 9HN, UK

${ }^{\mathrm{b}}$ Department of Cell Physiology and Pharmacology, University of Leicester, Lancaster Road, Leicester, LE1 9HN, UK

* Corresponding author. FAX: +441162525030.

Email address: rr18@le.ac.uk (Dr Rana El-Rachkidy) 


\begin{abstract}
Patch clamping was used to investigate, for the first time, the electrophysiological properties of pores formed by a cholesterol-dependent cytolysin in the membrane of nucleated cells rather than in artificial membranes as previously. Pneumolysin pores in inside-out patches of CHO cell membranes had a broad range of conductance classified into small $(<200$ $\mathrm{pS}$ ), medium ( $>200 \mathrm{pS}$ and $<1 \mathrm{nS}$ ) and large (>1 nS). A lytic-deficient mutant of pneumolysin (W433F) induced a similar spectrum of channels but there were more small channels than with wild-type (WT) toxin and the number of events was decreased. The WT toxin also induced channels when given to the inside surface of the plasma membrane.
\end{abstract}

Keywords: pneumolysin; patch clamp; pores; conductance 


\section{Introduction}

The pneumococcal toxin pneumolysin belongs to the family of cholesterol dependent cytolysins (CDCs). These toxins bind to cholesterol-containing membrane, oligomerise and generate large pores that disrupt the membrane integrity, leading to cell death [1]. Although these pores are formed in the membranes of all eukaryotic cells tested, their electrophysiological properties have been studied only with artificial membranes [2, 3]. In this work, the patch-clamp technique was used in inside out and outside out configurations to study, for the first time, the pores of one of the CDCs (pneumolysin) across the membrane of a nucleated cell.

In these experiments, we studied the lytic-deficient pneumolysin mutant, W433F, to investigate if lytic deficiency correlated with absence of electrophysiological properties. Trp433 is in a highly conserved region in all the CDCs is involved in the binding of the toxin monomers to the cell membrane [4].

We report that using the patch clamp technique a spectrum of different sized channels was observed in nucleated cells exposed to the WT pneumolysin but also in cells exposed to W433F pneumolysin. Interestingly, similar spectrum of channel sizes also was seen when the toxin was applied to the inside surface of the membrane.

\section{Material and Methods}

\section{Protein Purification}

WT pneumolysin was expressed and purified as before [5]. W433F mutant toxin was donated by Germie van den Dobbelsteen (NVI, The Netherlands). Purity and molecular weight $(53 \mathrm{kDa})$ of the toxins were analysed on $12 \%(\mathrm{w} / \mathrm{v})$ SDS-polyacrylamide gels. Haemolytic activity was assessed as before [6]. 
Patch Clamping of Chinese Hamster Ovary (CHO) cells

CHO cells were grown in MEM Alpha medium (Invitrogen) containing 10\% (v/v) foetal calf serum (FCS) in $100 \mathrm{ml}$ flasks. Cells were maintained at $37^{\circ} \mathrm{C}$ in $5 \%$ (v/v) carbon dioxide and were collected 30 minutes before use. The medium was removed and the cells were gently washed with PBS and then removed with Trypsin/EDTA. MEM Alpha was then added and the cell suspension was harvested and resuspended in $1 \mathrm{ml}$ MEM Alpha without FCS. The cell suspension was added into a $35 \mathrm{~mm}$ polylysine-coated culture dish, containing external bath solution and left for at least 20 minutes prior to patching.

\section{Recording Conditions}

Pneumolysin-induced channels were recorded from inside-out or outside-out patches excised from CHO cells. Pneumolysin was added to the pipette solution to a final concentration of $10 \mu \mathrm{g} / \mathrm{ml}$. Membrane currents were recorded with an Axopatch 200 amplifier (Axon Instruments, Foster City, CA, USA) connected to a PC for data acquisition and subsequent analysis. Patch-pipettes were made from thick walled, filamented borosilicate glass capillaries, which were coated with Sylgard and fire-polished to give resistances of 5-20 $\mathrm{M} \Omega$. The holding potential was set to $0 \mathrm{mV}$ and a $1.6 \mathrm{~s}$ pulse to +40 followed by a $1.6 \mathrm{~s}$ pulse to $-40 \mathrm{mV}$ was applied every second throughout the recordings. Membrane potentials are expressed as inside of the membrane relative to outside. For inside-out patches the pipette solution comprised (mM): $70 \mathrm{~K}^{+}, 70 \mathrm{Na}^{+}, 1 \mathrm{Ca}^{2+}$ and 10 HEPES, adjusted to pH 7.2 with $\mathrm{NaOH}$. The external (bath) solution comprised (mM): $140 \mathrm{~K}^{+}, 10$ EDTA, 10 HEPES and the $\mathrm{pH}$ was adjusted to 7.2 with $\mathrm{KOH}$. For experiments using outside-out patches, these solutions were reversed. All recordings were made at room temperature $\left(20-22^{\circ} \mathrm{C}\right)$. 


\section{Results}

\section{Experiment optimisation}

CHO cells were used as they have few intrinsic channels in their plasma membrane. Control recordings of up to 60 min duration, in the absence of toxin, confirmed quiescence (Fig. 1Aa). Different concentrations of the WT toxin, from 2 to $100 \mu \mathrm{g} / \mathrm{ml}$, were included in the pipette solution. Channel openings with $2 \mu \mathrm{g} / \mathrm{ml}$ toxin took several minutes to appear, while with $50 \mu \mathrm{g} / \mathrm{ml}$ the formation of pneumolysin channels was too rapid to allow appropriate measurements and analysis. A concentration of $10 \mu \mathrm{g} / \mathrm{ml}$ toxin was judged appropriate for further experiments

\section{Inside-out patches}

We first tested the ability of pneumolysin to form pores when applied to the outside surface of CHO. Typical recordings from an inside-out patch are shown in Fig. 1A. After patch formation, openings and closings of channels with different conductances were observed. These were attributed to pneumolysin because such channels were never seen in control patches obtained in the absence of pneumolysin (Fig. 1Aa). Opening events could be classified into three groups according to their conductances: 'small' ( $<200$ pS; Fig. 1Ab), 'medium' (>200 pS and <1 nS; Fig. 1Ac) and 'large' (>1 nS; Fig. 1Ad). Conductance was calculated using a reversal potential for the pores of $0 \mathrm{mV}$ as the currents at +40 and $-40 \mathrm{mV}$ were of approximately equal amplitude but opposite direction. The frequency of occurrence of small, medium and large channels in inside-out patches is shown in Table 1. Medium channels were observed mostly, with a predominant conductance around $700 \mathrm{pS}$. Low conductance channels were seen much less often than medium channels and generally these small channels were open for a short period of time only. In contrast to small and medium channels, large channels were open for a longer duration. Because of the complex behaviour 
and multiple conductances of pneumolysin-induced channels, it was not possible to obtain a quantitative measure of the open-time distribution of the various conductance states.

The mean conductance for each $+40 \mathrm{mV}$ pulse was plotted against time, as shown in Fig. 1B. These data are representative of 15 other patches. In control patches, in the absence of toxin, no increase in membrane conductance was detected (Fig. 1B (a)). However, when pneumolysin was added to the patch pipette an increase in membrane conductance was observed, as shown in Fig. 1B (b-d). Generally, the pattern seen in Fig. 1B (b) was observed, with a low mean conductance at the beginning, followed by stepwise increases in the mean membrane conductance with time. However, in some recordings large mean conductance states were followed by a rapid, large decrease in the membrane conductance, as indicated by arrows in Fig. 1B (c-d). An average of the conductance change across the membrane from 5 inside-out patches is shown in Fig 1C.

\section{Outside-out patches}

To exploit the versatility of patch clamping, outside-out patches were obtained with pneumolysin in the pipette solution. Outside-out patches remained quiescent in the absence of pneumolysin. As Fig 2A illustrates, pneumolysin formed channels when in contact with the cytosolic side of the cell membrane. The conductance properties of the channels were similar to those formed when the toxin was on the external side of the membrane. Similar results were seen in four other outside-out patches. These experiments show that pneumolysin induced small medium and large channels when applied to the internal side of the membrane. The frequency of these channels is shown in Table 1. As can be seen, the frequency of occurrence of each type in outside-out patches is approximately the same as with inside-out patches.

How conductance changed with time can be seen in Fig. 2B. As with inside-out patches, conductance was generally low at the beginning but gradually increased with time 
(Fig. 2Ba). Sometimes large conductance states occurred within the recordings and were followed by a rapid decrease in conductance, as indicated by the arrow in Fig. 2Bb.

\section{Channels induced by W433F}

To test if patch clamping could differentiate the channel properties of a mutated CDC, the virtually lytic deficient mutant W433F, was tested in inside-out patches. Fig. 3A shows a typical recording of the channels that were induced. The amplitudes of channels by W433F could also be classed as small, medium and large (Fig. 3). However, the relative frequency of channels induced by W433F differed from the WT toxin (Table 1). There was a large increase in the proportion of small channels, while the frequency of both medium and large channels was lower. However, medium conductance channels, with a predominant conductance of around $700 \mathrm{pS}$, were still the most prevalent. The change in the mean conductance with time is shown in Fig. 3B. As was seen with the WT toxin, the conductance due to W433F-induced channels increased with time but the rate of increase was much slower, with a notable increase observed only in patch c. Interestingly, in contrast to WT, the closure of large conductance channels was not observed (Fig 3Bc). The membrane patch was stable, with the large channel remaining open. Only very rarely was disruption of the patch seen with mutant toxin. A comparison of the increase in conductance with time, averaged from 3 and 5 patches for the mutant and WT toxin is shown in Fig 3C. Less events were detected with W433F toxin than with WT. For instance, as shown in Fig 4, with the WT toxin, 573 events were recorded in 30 min, whereas for W433F only 155 events were detected over 74 min.

It is noteworthy that CHO cells near the tip of the patch pipette were not affected by contamination of the bath with the W433F toxin, even after 1hr exposure, whereas cells near the patch pipettes with the WT toxin died after a few patches. 


\section{Discussion}

This paper reports the first description of the electrophysiology of the channels formed by cholesterol-dependent cytolysins in a natural biological membrane. Until now, electrophysiology of these channels has been studied only in planar lipid bilayers. Although work with these model systems has provided valuable information, the data have lacked the validation that come from experiments with natural membranes, with their inherently greater complexity. Here we show that patch clamping can be used to correct this omission.

The data we obtained contribute to a debate with the CDC research community. In 2000, Shepard and colleagues [7] reported only events of a single conductance in lipid bilayers exposed to perfringolysin. They suggested that only a single conductance state supported the hypothesis that the formation of a prepore made up of a full ring oligomer must assemble prior to its insertion in the membrane and channel formation. Recently, using cryoelectron microscopy, Tilley and colleagues [1] demonstrated conclusively that pneumolysin forms a prepore. However, combination of the data in this paper, with the cryo-electron microscopy shows that a prepore complex and multiple conductance channels coexist. Our data validate previous lipid bilayer experiments that observed that a range of conductance channels was seen with pneumolysin [3] and perfringolysin [2].

The difference in the observations on conductance channels obtained in this work and Shepard's, could be due to differences in the channel behaviour of the two toxins, but it seems that is more likely to be due to technical variations, considering that the same pattern of channels seen with pneumolysin was obtained elsewhere with perfringolysin in planar lipid bilayer [2].

One explanation for the different conductance channels is that they reflect different physical sizes of oligomers inserting into the membrane. This seems unlikely. Many studies have shown the presence of arc and ring structures in membrane exposed to CDCs [8-10], but 
provided no evidence that complete oligomers of CDCs (rings) of greatly differing sizes enter the membrane. The arcs are believed by some to be membrane-inserted but incomplete oligomers that can function as channels [10-12]. If so the different conductance states may be due to the physical differences in the arcs and ring structures.

An alternative possibility is that the membrane-inserted pneumolysin oligomer is not simply forming a static vertical transmembrane column of water commensurate with an oligomer diameter of around 30nm, but has a more complex and/or dynamic internal structure. Variation in the internal structure may give rise to channels of differing conductance and also explain how the channels open and close, without apparent changes in the external appearance of the channel. Here we show that a single amino acid change in W433F can alter the distribution of conductance states and the behaviour of channels even though previous electron microscopy found the apparent diameter of the W433F oligomers were not different from the wild-type but channels formed by this mutated toxin in lipid bilayer had altered cation sensitivity [3].

The possibilities provided by patch clamping to investigate interactions with the inside, as well as the outside of membranes (through inside-out and outside-out configurations) led us to a new finding that is not possible with artificial systems. Namely, pneumolysin can form channels when applied to either side of the membrane. The finding that pneumolysin interacts with the membrane on both sides rules out the necessity of a protein receptor specifically on the outer leaflet of the membrane. Although Hiyashi and his colleagues in [13] showed that a toxin from the CDC family, perfringolysin, can bind cholesterol-rich domains in the inner leaflet of the membrane, our work shows the first recordings of pores generated by a CDC when applied to the inner side of the membrane.

To date, recordings of the pores of pore-forming toxins in 'real' cell membranes have not been fully explored and, until this study, has not been done with any of the CDCs. Questions remain on the mechanism of channel functioning (opening and closing) and the 
molecular mechanism behind the heterogeneity of the conductances. This study shows that the patch clamp technique can be more exploited to examine these issues in the environment of a living cell. 


\section{Figure Legends}

Fig.1. Pneumolysin-induced channels across inside-out patches of the membrane of CHO cells.

A. Recordings from inside-out patches, at the membrane potentials indicated by the voltage schedule shown, in the absence (a) and presence of pneumolysin (b-d). Small, s, medium, m, and large, l. channels are indicated by the arrows.

B. Change over time, of the mean conductance $(\mathrm{G})$ of the membrane due to pneumolysin induced-channels in the absence (a) and presence of pneumolysin (b-d).

C. Conductance change averaged from 5 inside-out patches.

Fig. 2. Pneumolysin-induced channels across outside-out patches of CHO cells.

A. Recordings at the membrane potentials indicated by the voltage schedule above, (a) small, s, (b) medium, m and (c) large, l, conductance channels are indicated with arrows.

B. Increase of the mean conductance of the membrane due to pneumolysin-induced channels, in two outside-out patches.

Fig. 3. W433F-induced channels in inside-out patches of $\mathrm{CHO}$ cells.

A. Small, s, medium, m and large, l, channels observed in an inside-out patch with $10 \mu \mathrm{g} / \mathrm{ml}$ of W433F pneumolysin.

B. Plot of the mean conductance against time for three inside-out patches.

C. The solid line shows the conductance against time averaged from 3 patches with W433F. For comparison, the dashed line are the data of Fig 1C with WT toxin in the pipette.

Fig. 4. Distribution of conductance events in two inside-out patches.

All events detected in each recording were collated and binned (50 pA wide). There were 573 events in 30 minutes for WT and 87 events in 74 minutes for the W433F mutant. 


\section{References}

[1] S.J. Tilley, E.V. Orlova, R.J. Gilbert, P.W. Andrew, H.R. Saibil, Structural basis of pore formation by the bacterial toxin pneumolysin, Cell 121 (2005) 247-256.

[2] G. Menestrina, C.L. Bashford, C.A. Pasternak, Pore-forming toxins: experiments with S. aureus alpha-toxin, C. perfringens theta-toxin and E. coli haemolysin in lipid bilayers, liposomes and intact cells, Toxicon 28 (1990) 477-491.

[3] Y.E. Korchev, C.L. Bashford, C. Pederzolli, C.A. Pasternak, P.J. Morgan, P.W. Andrew, T.J. Mitchell, Conserved tryptophan in pneumolysin is a determinant of the characteristics of channels formed by pneumolysin in cells and planar lipid bilayers, Biochem J. 329 (1998) 571-577.

[4] H. Baba, I. Kawamura, C. Kohda, T. Nomura, Y. Ito, T. Kimoto, I. Watanabe, S. Ichiyama, M. Mitsuyama, Essential role of domain 4 of pneumolysin from Streptococcus pneumoniae in cytolytic activity as determined by truncated proteins, Biochem Biophys Res Commun. 281 (2001) 37-44.

[5] R.J. Gilbert, J. Rossjohn, M.W. Parker, R.K. Tweten, P.J. Morgan, T.J. Mitchell, N. Errington, A.J. Rowe, P.W. Andrew, O. Byron, Self-interaction of pneumolysin, the poreforming protein toxin of Streptococcus pneumoniae. J Mol Biol. 284 (1998) 1223-1237.

[6] R.H. Owen, G.J. Boulnois, P.W. Andrew, T.J. Mitchell, A role in cell binding for the Cterminus of pneumolysin, the thiol-activated toxin of Streptococcus pneumoniae. FEMS Microbiol Lett. 121 (1994) 217-221.

[7] L.A. Shepard, O. Shatursky, A.E. Johnson, R.K. Tweten, The mechanism of pore assembly for a cholesterol-dependent cytolysin: formation of a large prepore complex precedes the insertion of the transmembrane beta-hairpins, Biochemistry 39 (2000) 1028410293. 
[8] P.J. Morgan, S.C. Hyman, A.J. Rowe, T.J. Mitchell, P.W. Andrew, H.R. Saibil, Subunit organisation and symmetry of pore-forming, oligomeric pneumolysin, FEBS Lett. 371 (1995) 77-80.

[9] P.J. Morgan, S.C. Hyman, O. Byron, P.W. Andrew, T.J. Mitchell, A.J. Rowe, Modeling the bacterial protein toxin, pneumolysin, in its monomeric and oligomeric form, J Biol Chem. 269 (1994) 25315-25320.

[10] M. Palmer, R. Harris, C. Freytag, M. Kehoe, J. Tranum-Jensen, S. Bhakdi, Assembly mechanism of the oligomeric streptolysin O pore: the early membrane lesion is lined by a free edge of the lipid membrane and is extended gradually during oligomerization, EMBO J. 17 (1998) 1598-1605.

[11] R.J. Gilbert, Pore-forming toxins, Cell Mol Life Sci. 59 (2002) 832-844.

[12] R.J. Gilbert, Inactivation and activity of cholesterol-dependent cytolysins: what structural studies tell us, Structure 13 (2005) 1097-1106.

[13] M. Hayashi, Y. Shimada, M. Inomata, Y. Ohno-Iwashita, Detection of cholesterol-rich microdomains in the inner leaflet of the plasma membrane, Biochem Biophys Res Commun. 351 (2006) 713-718. 
Fig. 1.

A

$+40 \mathrm{mV}$

a

b

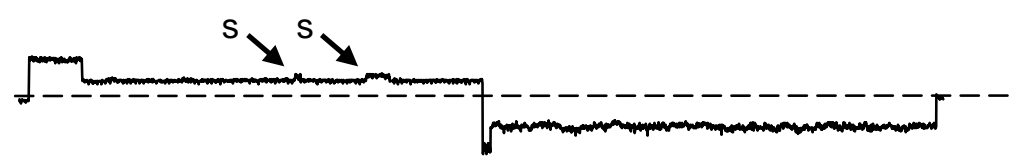

C

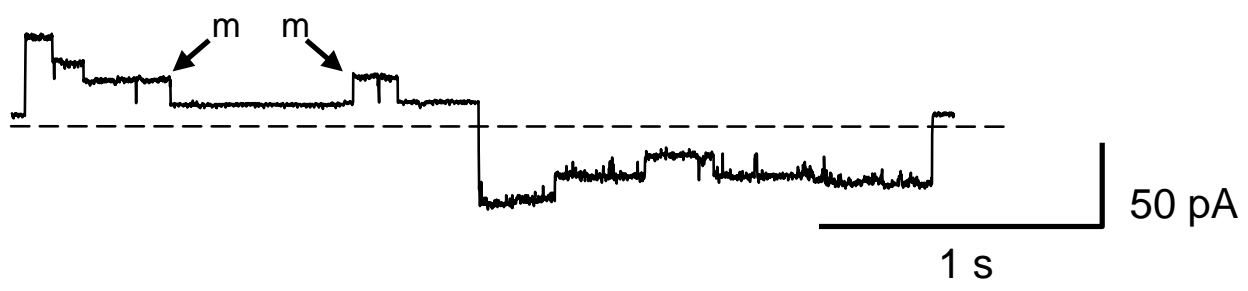

d

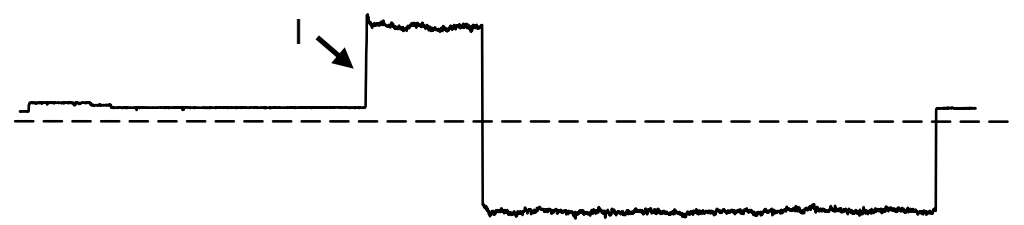

B
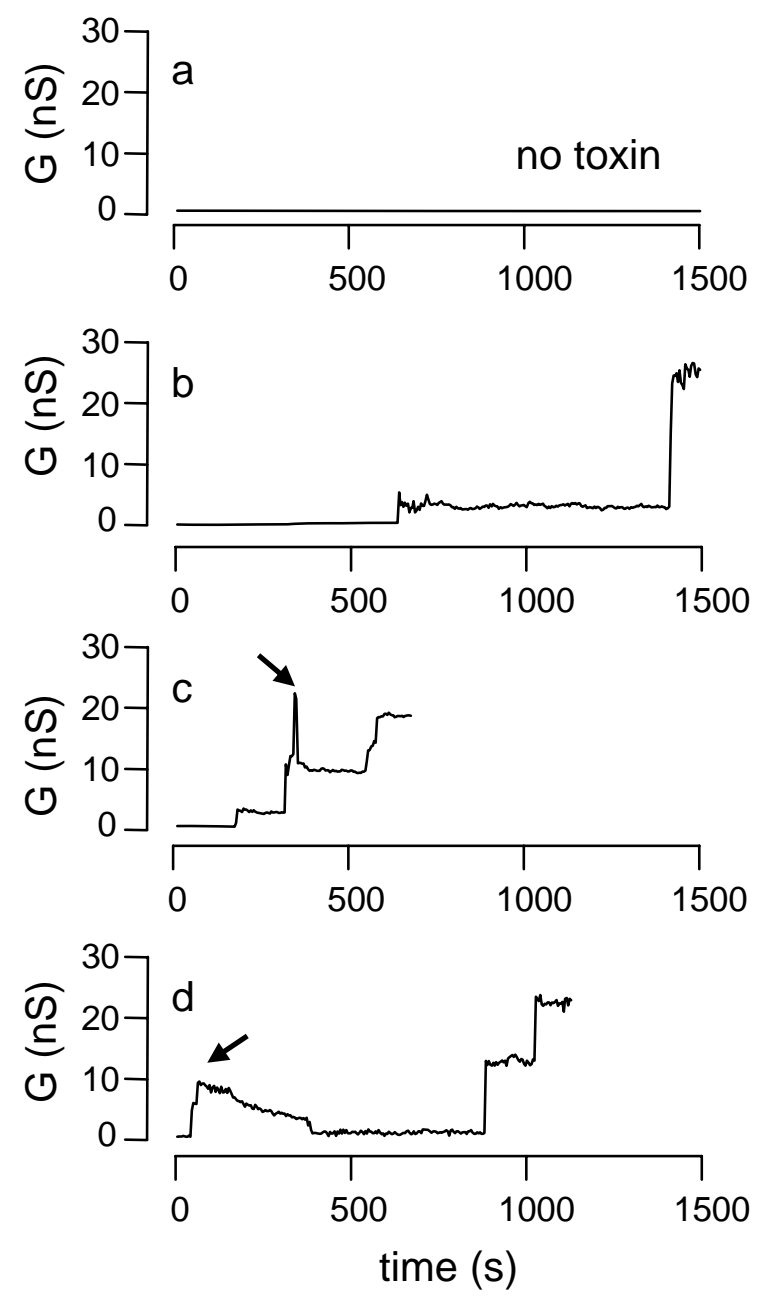

no toxin 500 pA

C

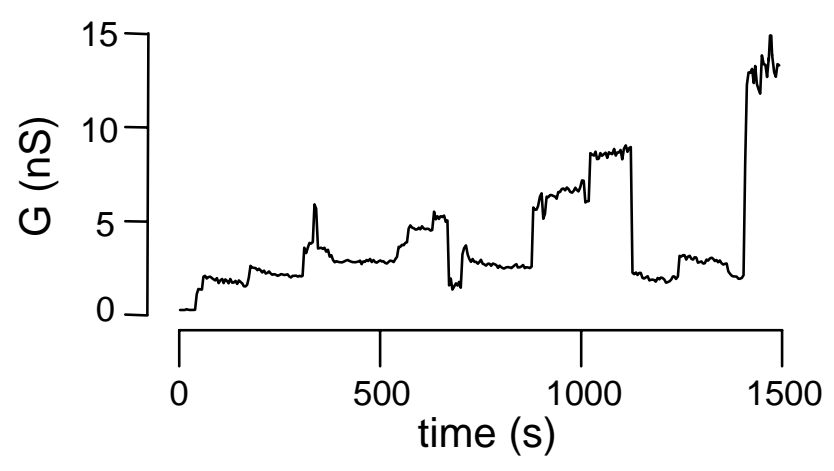


Fig. 2.
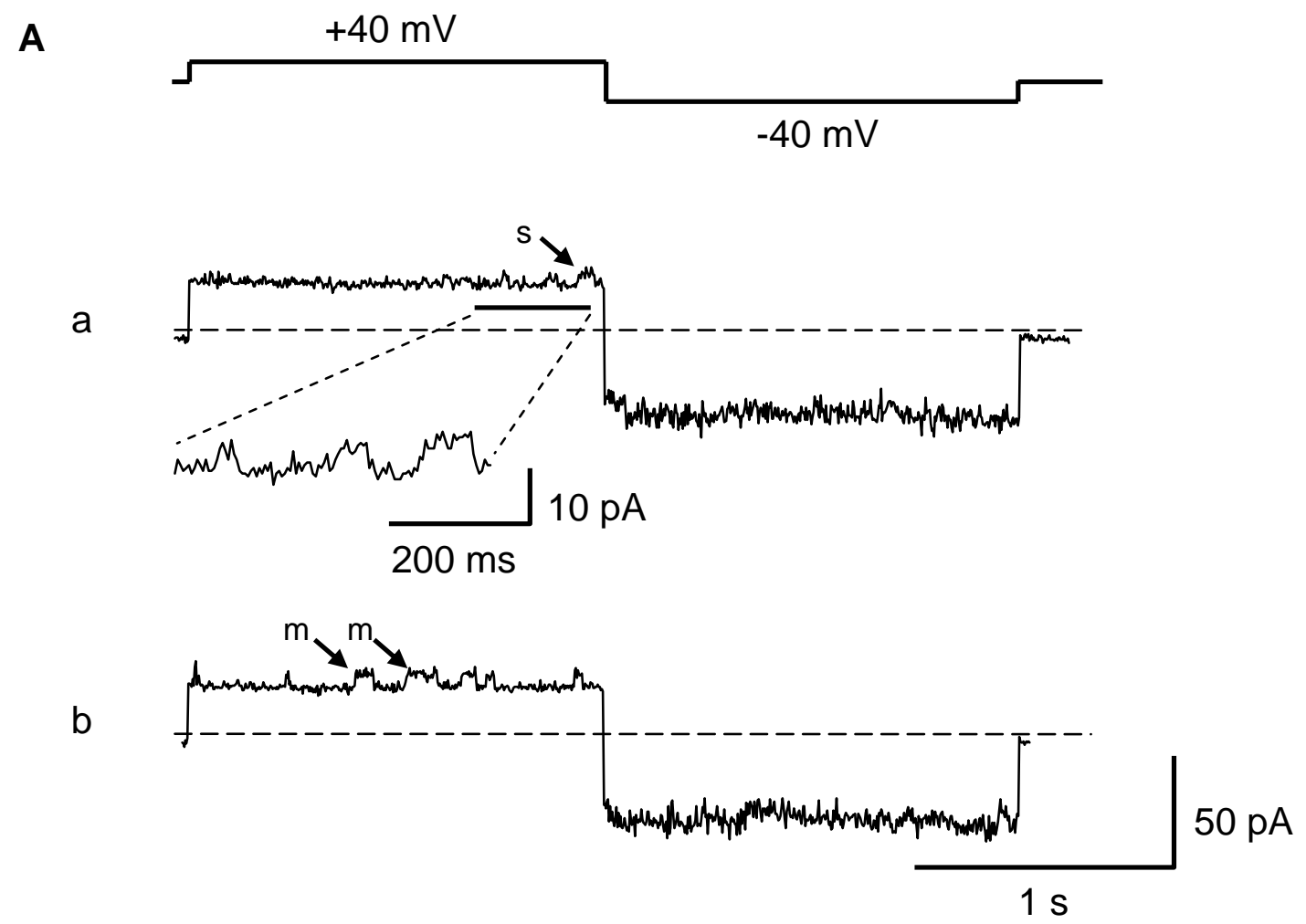

C

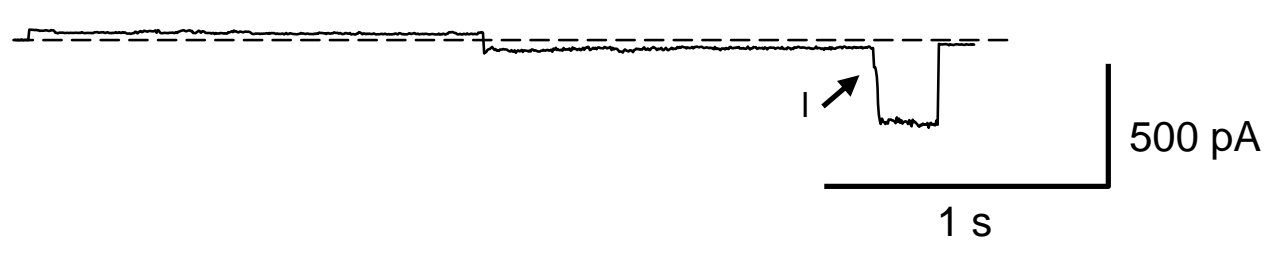

B
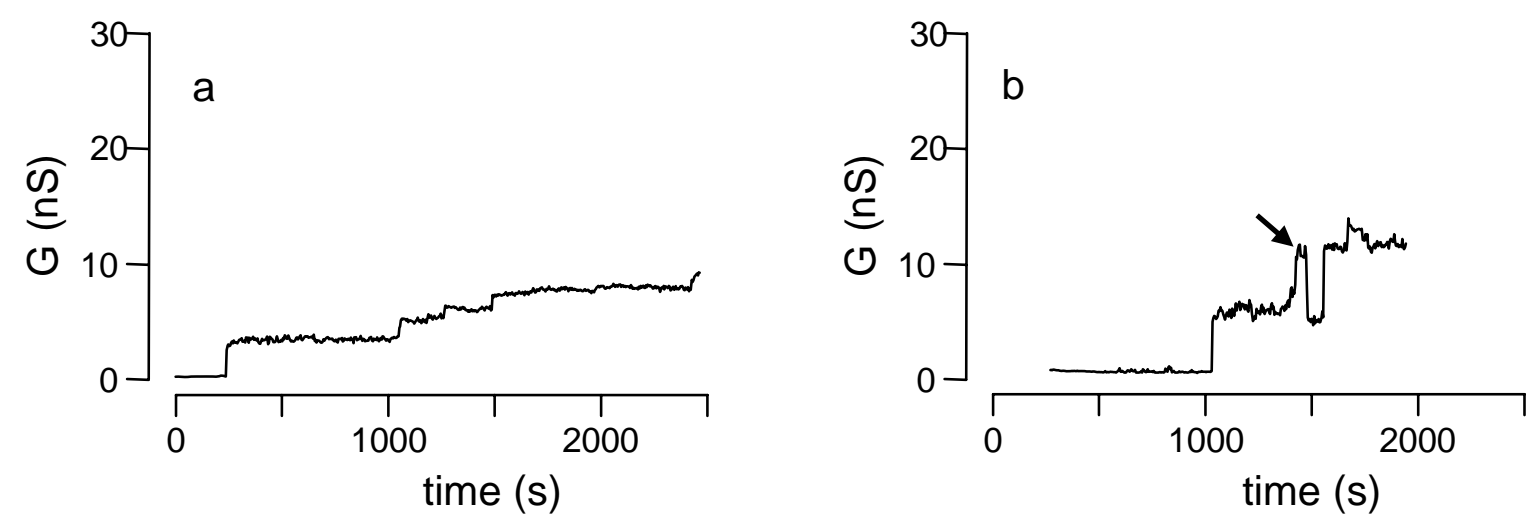
Fig. 3.

A
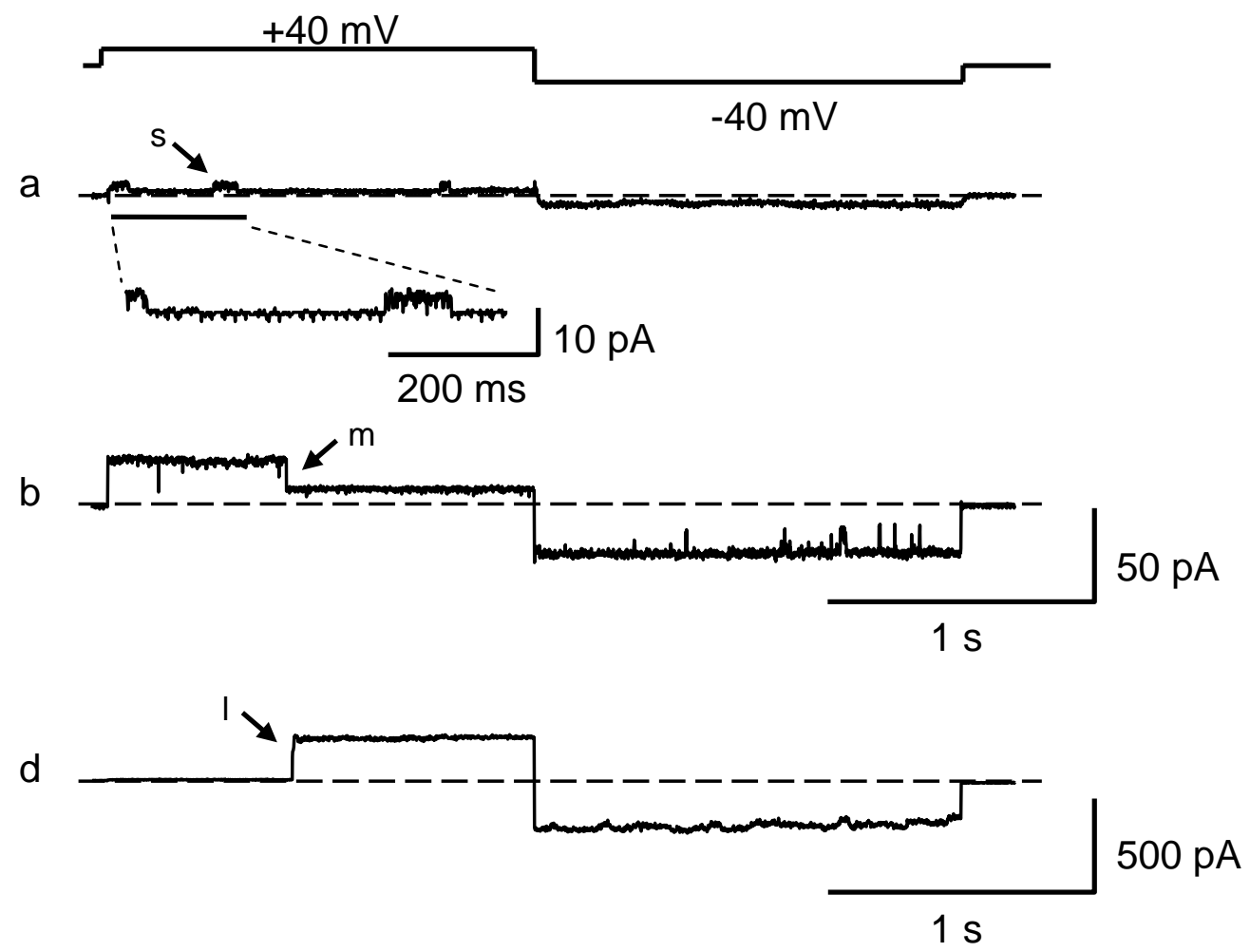

B
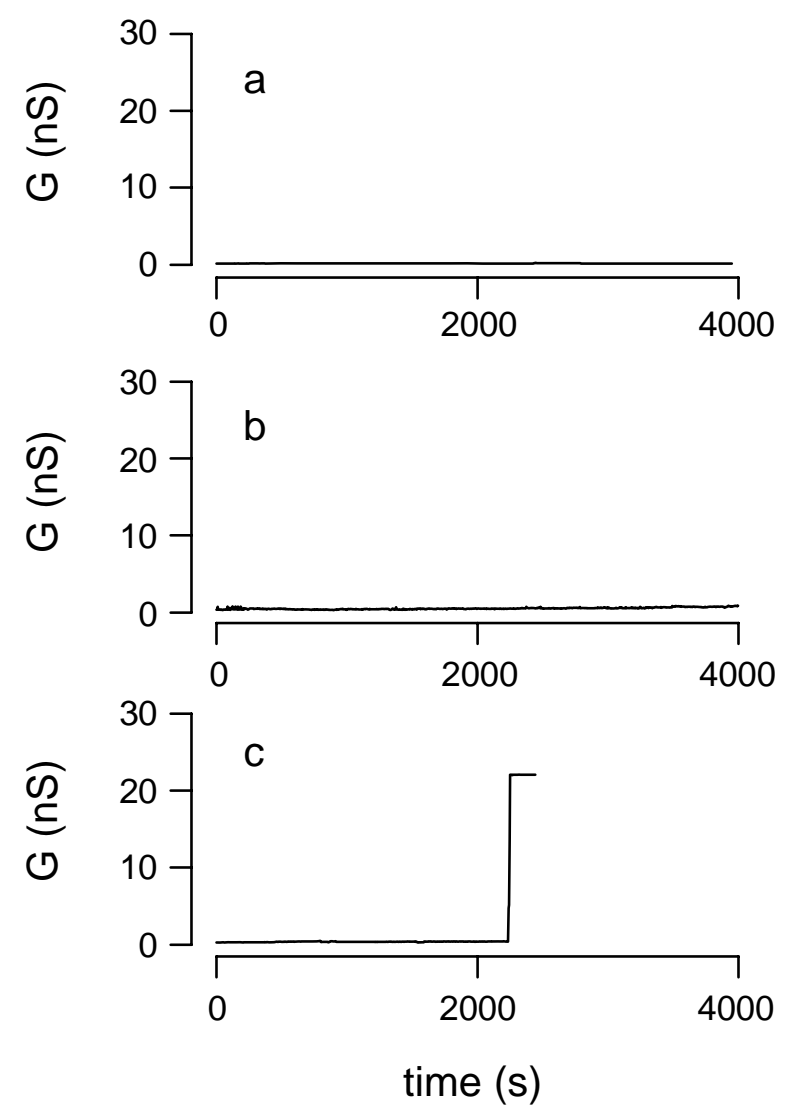

C

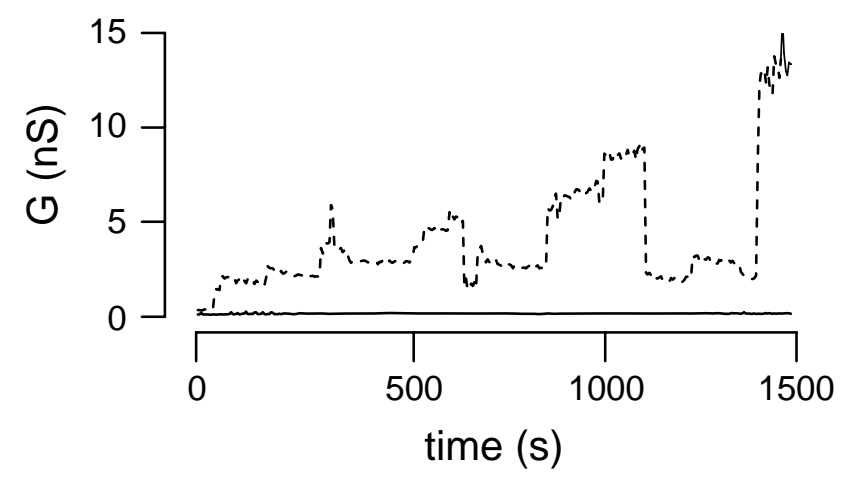


Fig. 4.

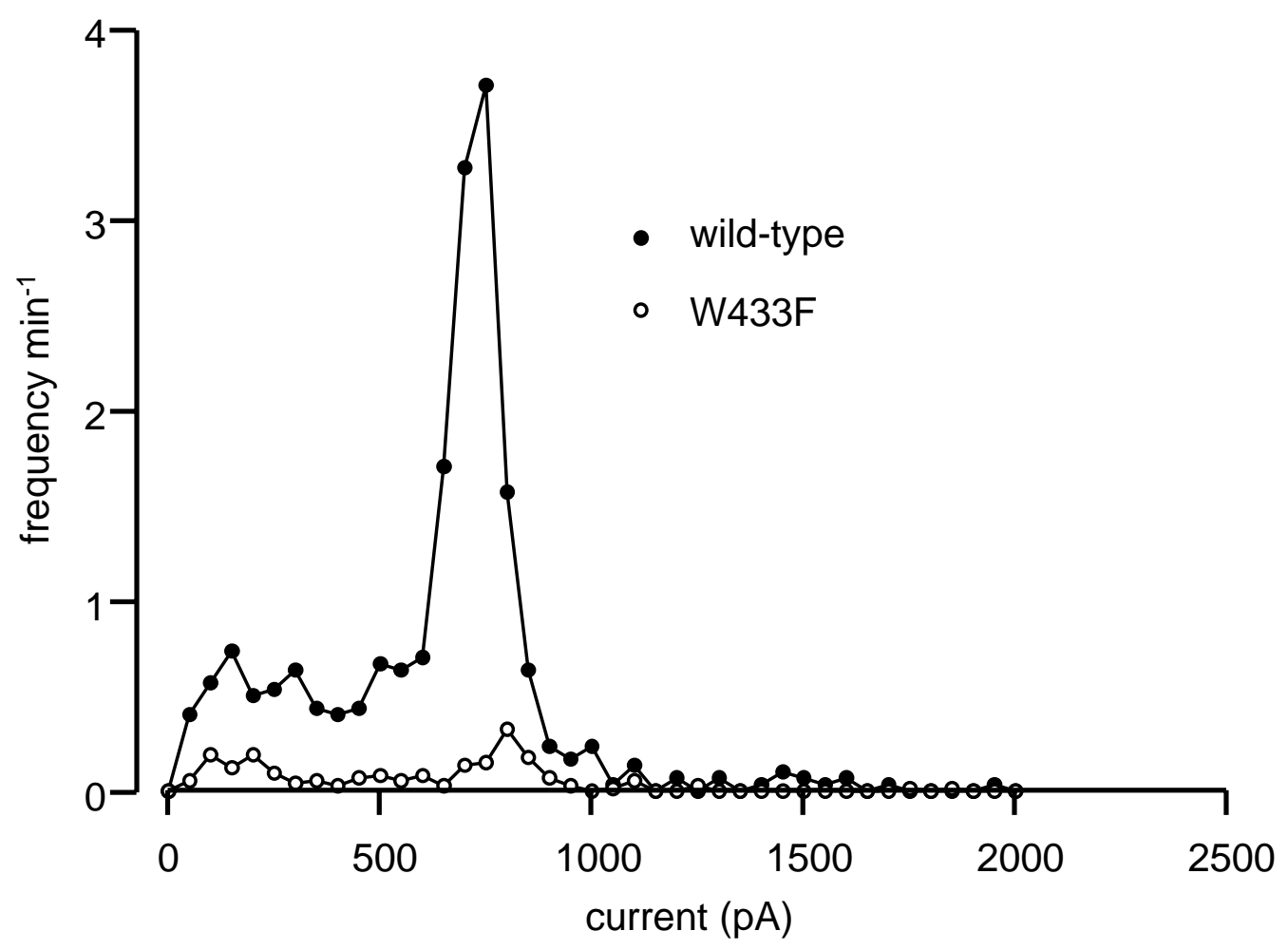


Table 1. Frequency of small, medium and large channels of wild-type (WT) pneumolysin and W433F mutant within a time frame of 20 minutes

\begin{tabular}{|c|c|c|c|}
\hline & \multicolumn{3}{|c|}{ Relative Frequency } \\
\hline Channel size & $\begin{array}{c}\text { WT PLY } \\
\text { (inside-out) }\end{array}$ & $\begin{array}{c}\text { WT PLY } \\
\text { (outside-out) }\end{array}$ & $\begin{array}{c}\text { W433F PLY } \\
\text { (inside-out) }\end{array}$ \\
\hline Small $(<200 \mathrm{pS})$ & 11 & 6 & 28 \\
\hline Medium $(>200 \mathrm{pS}-<1 \mathrm{nS})$ & 84 & 89 & 72 \\
\hline Large $(>1 \mathrm{nS})$ & 5 & 5 & 0 \\
\hline
\end{tabular}

